'Departamento de Trabajo Social de la Facultad de Ciencias

Sociales de la Universidad de Concepción, Concepción, Chile

Tatrabajadora Social, Universidad de Concepción.

${ }^{\mathrm{b}}$ Magister en Trabajo Social y Políticas Sociales.

'Magister en Salud Pública y Gestión Sanitaria.

${ }^{d}$ Doctora en Sociología

Trabajo no recibió financiamiento Las autoras declaran no tener conflictos de interés.

Recibido el 19 de marzo de 2020, aceptado el 22 de enero de 2021.

Correspondencia a: Carla González Sanzana: carla.gonzalez.sanzana20@ gmail.com

\section{Desafíos de la calidad percibida y el ejercicio del derecho a la información de los/as usuarios/as de las unidades de urgencias hospitalarias}

\author{
CARLA GONZÁLEZ SANZANA ${ }^{1, \mathrm{a}, \mathrm{b}}$, CECILIA BUSTOS IBARRA $^{1, \mathrm{a}, \mathrm{b}, \mathrm{c}, \mathrm{d}}$
}

Background: Communication gaps are common in emergency medicine. Aim: To know how the health information was delivered to users and relatives by the health care team in the Emergency Unit of a general hospital and how the standards dictated by the patients' rights and duties law were complied with. Material and Methods: Health care workers, patients and their relatives were subjected to semi-structured interviews, and their behavior was directly observed. Results: Important gaps that violate the right to information of users and family members were detected. There is a paucity of minimal conditions to protect the confidentiality and privacy of the information about diagnosis, treatment, or prognosis. There is no time allocated to deliver information and there are no physical spaces for such purpose. Conclusions: The health care team is not trained in communication skills, crisis intervention and empathy. Empathy and good communication are essential for users and family members to perceive health care as satisfactory and safe and to comply with indications. The lack of protocols to inform family members about the death of a patient is the finding that most clearly accounts for the observed communication deficiencies.

(Rev Med Chile 2021; 149: 366-377)

Key words: Emergency Medicine; Health Communication; Right to Health.

E 1 ejercicio del derecho a la salud en Chile presenta importantes brechas que han sido suficientemente documentadas por diversos autores/as y organismos nacionales e internacionales tales como INDH (2016), OCDE (2014) y OMS (2017). El Ministerio de Salud elaboró las "Orientaciones Técnicas para el Rediseño del Proceso de Atención de Urgencia de Adulto en las Unidades de Emergencia Hospitalaria", detectando los nodos críticos relacionados a distintas situaciones que convergen en Unidades de Urgencia Hospitalaria (en adelante UUH), que dan cuenta de una débil pertinencia en las consultas y aumento de las hospitalizaciones en los últimos 5 años (7,7\% a 9,7\%), atribuido a la complejidad del motivo de consulta, sumando aquellas dificultades propias del acceso a la información (MINSAL, 2018). La Encuesta de Atención al Usuario/a en UUH, señaló como principal dificultad débil comprensión de las indicaciones médicas de usuarios/ as con alta médica (MINSAL, 2018).

A nivel de normativa nacional, la Ley 20.584, "Ley de Derechos y Deberes que Tienen las Personas en Relación con Acciones Vinculadas a su Atención de Salud", en su Artículo 5 señala "todo usuario tiene derecho a ser tratado con gentile- 
za y respeto, obteniendo información sobre su estado de salud de manera clara, comprensible $\mathrm{y}$ sin dificultad en un ambiente que proteja su dignidad" (BCN, 2012). Para proteger el derecho a la información respecto al diagnóstico, tratamiento y pronóstico, tal información puede ser proporcionada al mismo/a usuario/a, familiar o representante legal. Esta ley indica que el/la médico/a es el/la principal responsable de otorgar información oportunamente sobre diagnóstico tentativo, alternativas de tratamiento y posible pronóstico (Ley No 20.584, 2012). Este trabajo busca identificar las brechas en el plano de la comunicación entre el equipo de salud y los/as usuarios/as-familiares en torno a la entrega de información en una UUH de un Hospital Público de Alta Complejidad. El marco teórico consideró el Modelo Tridimensional de la Comunicación médico-paciente, Enfoque de Derecho en Salud, Modelo de Calidad en Salud y el Modelo Biomédico versus Biopsicosocial. El marco conceptual desarrolló los conceptos de Satisfacción Usuaria y Calidad de Atención, mientras que el marco empírico estuvo compuesto por nueve investigaciones nacionales e internacionales.

La pregunta de investigación ¿La información otorgada sobre diagnóstico y tratamiento a los/as usuarios/as y familiares de las UUH cumple con los estándares del derecho a la información establecidos en la Ley 20.584? Objetivo general conocer cómo la información sobre diagnóstico y tratamiento a usuarios/as cumple con los estándares de la ley de derechos y deberes 20.584 en la relación al equipo de salud-usuario/a-familiares. Objetivos específicos fueron conocer la satisfacción de usuarios/as y familiares frente la información recibida en UUH y conocer la percepción del equipo de salud de la UUH frente la información entregada. Respecto la revisión teórica y empírica, el diseño consideró cinco supuestos preliminares: 1) La calidad de la información obtenida en la UUH en torno a diagnóstico y tratamiento presenta falencias relacionadas a su contenido, el que es poco claro, utilizando tecnicismos poco comprensibles para el/la usuario/a-familiar; 2) Los canales de información orales y escritos entre funcionario/a-usuario/a-familiar como consentimiento informado, epicrisis, ficha clínica y receta no proporcionan la información de manera acabada para que el/ la usuarios/a-familiar comprenda a cabalidad su diagnóstico o tratamiento; 3) El equipo de salud no cuenta con las habilidades comunicativas suficientes para el traspaso efectivo de la información a usuarios/as-familiares; 4) El equipo de salud no dispone de un espacio físico apto para establecer la entrega de información a los/as usuarios/ as-familiares, resguardando su confidencialidad y privacidad y 5) El equipo de salud no dispone de tiempo suficiente para traspasar la información de diagnóstico y tratamiento a usuarios/as-familiares.

\section{Material y Método}

La metodología utilizada fue Cualitativa, con un enfoque Fenomenológico centrada en profundizar en el fenómeno desde la experiencia de sus propios protagonistas (Tojar, 2006). Estudio de tipo descriptivo..

El contexto seleccionado para desarrollar la investigación fue la UUH del Hospital Guillermo Grant Benavente (en adelante HGGB), ubicado en la ciudad de Concepción, VIII Región del Bío Bío, Chile. El MINSAL (2019) señala que según una medición latinoamericana el HGGB ocupó el primer lugar como mejor equipado en el ranking HospiRank, frente a recintos de salud de ocho países.

La investigación fue aprobada por el Comité de Ética Científico del HGGB quien autorizó la realización de la investigación el 13.08.2019.

El diseño muestral utilizado correspondió al Muestreo Intencional, indicando que en la investigación deben incluir en el grupo de informantes aquellos que intervienen directamente con el fenómeno estudiado, presumiendo que podrán proporcionar insumos más enriquecedores (Tojar, 2006).

La muestra estuvo compuesta por tres perfiles de Informantes: funcionarios/as de salud, usuarios/as y familiares o referente significativo. Los Criterios de Inclusión para Perfil Funcionarios/as fue tener como mínimo dos años de experiencia en la UUH Adulto, desempeñarse en una función que incluyera el trato directo con usuarios/as. Respecto a los/as usuarios/as debían presentar una situación de urgencia catalogadas en grados $\mathrm{C} 2$ o $\mathrm{C} 3$, pertinentes de ser atendidas en este servicio. Además, se consideraron a usuarios/as hospitalizados/as, ello con el fin de poder observar la interacción entre las partes por mayor tiempo.

Los familiares eran parientes directos, que 
podían expresarse sin dificultad, no presentaban discapacidad mental y se encontraban en la UUH o en la sala de espera. Estos/as familiares, si bien habían recibido información, ésta no era clara y no resolvía completamente sus dudas, pero habían familiares que a pesar de sus intentos, no lograron entrevistarse con el/la médico/a.

Las técnicas de recolección de información utilizadas fueron: Entrevista Semiestructurada y Observación Participante. Por razones de factibilidad, se definió realizar 5 entrevistas a cada perfil, alcanzando un total de 15 entrevistas, utilizando una pauta guía para cada perfil. Para efectos de contacto y selección de los informantes se utilizó una Ficha de Selección de Participantes y para garantizar la calidad de los datos obtenidos y contribuir a la reflexividad del proceso de investigación se utilizó una Ficha de Control de Calidad. Previo inicio del proceso de entrevistas se dio a conocer a los/as participantes un Consentimiento Informado el cual el/la entrevistado/a leyó, recibiendo una copia al momento de ser firmado. Las entrevistas fueron realizadas (previo acuerdo con la persona entrevistada) en espacios físicos de la UUH, como la oficina de Entrevista Familiar y Sala AUGE, disponibles en el momento en que se desarrollaron las entrevistas, las que tuvieron un tiempo de duración entre los 30 a 45 minutos, fueron grabadas y posteriormente trascritas literalmente utilizando las Normas de Jefferson, tareas de responsabilidad de la investigadora principal, con el propósito de velar por la calidad de las transcripciones, que permitieron revivir las instancias de entrevistas y nutrir mayormente los escritos (Verd y Lozares, 2016).

Todas las entrevistas fueron realizadas de lunes a viernes a partir de las 08:00 a las 17:00 hrs., pues era necesario contar con la supervisión del tutor Asistente Social de la UUH, por tanto, para efectos de las entrevistas a funcionarios/as y usuarios/as se realizaron en dichos horarios, mientras que las entrevistas a familiares se aprovechó instancia de horario de visita a usuarios/as para realizarlas, es decir, desde las 13:00 a 14:00 hrs. Por tanto, los resultados de las entrevistas fueron recogidas en horario diurno y no permiten captar posibles diferencias en función de su horario, cuestión que si se logra mediante la técnica de Observación Participante.

La Observación Participante, estuvo centrada en las interacciones entre los sujetos que con- forman la muestra, utilizando una Pauta Guía de Observación que consideraba los siguientes elementos: el comportamiento del/la participante frente la información recibida o entregada, lenguaje no verbal, los medios o canales de comunicación utilizados, el espacio físico. Esta Pauta Guía se basó en categorías de observación lo que propició un registro de la información levantada, orientada a recoger con mayor profundidad y riqueza las notas plasmadas (Verd y Lozares, 2016). Fue realizada en cuatro distintos días y horarios. El primer momento fue día hábil horario diurno, en UUH Adulto, segundo momento día hábil horario nocturno en UUH Pediátrica, tercer momento día de fin de semana horario madrugada en sala de espera de UUH adulto y cuarto momento día de fin de semana horario diurno en sala de espera UUH Pediátrica.

El análisis realizado corresponde al Análisis de Contenido, con el apoyo del software Cualitativo Atlas. Ti.

\section{Resultados}

Los principales resultados señalan que existe una importante vulneración al derecho a la información hacia usuarios/as y familiares. Aquel hallazgo se puede develar al comprobar que el contexto de la UUH del HGGB no cuenta con espacios propicios para entregar la información de manera adecuada y aquellos espacios que se utilizan en la actualidad no permiten garantizar la privacidad y confidencialidad de la información. En la actualidad se utilizan el sector de mesones, pasillos, sala de espera, al lado de la camilla y sector de hidratación para realizar esta entrega de información. Sin embargo, hay antecedentes anteriores que indican que se creó una oficina para entregar información, no obstante, nunca se utilizó para dicha función, sino que se utilizó para hospitalizaciones. Otro resultado relevante es la constatación de que la entrega de información a los usuarios, no constituye una prioridad en la UUH, puesto que no existen instancias de capacitación o formación para el equipo de salud en torno a la entrega de información, por lo cual ésta es entregada según los criterios personales de cada funcionario/a, que según nuestros hallazgos no corresponden a información completa. Tampoco existen instancias que permitan potenciar o de- 
Tabla 1. Resumen de resultados observación participante

\begin{tabular}{|c|c|}
\hline Categorías & Resúmenes de las observaciones \\
\hline Tipo de interacción & $\begin{array}{l}\text { La más observadas fueron las de guardias de seguridad y el Asistente Social con familiares. } \\
\text { La función de los guardias de seguridad es significativa en el sector de la sala de espera } \\
\text { tanto de la unidad pediátrica/obstétrica y en la unidad de adulto, ya que está en constante } \\
\text { interacción con los familiares al igual que el Asistente Social. En menos medida se observa- } \\
\text { ron interacciones de enfermeros/as y TENS con familiares que solicitaban información de las } \\
\text { mismas características. }\end{array}$ \\
\hline $\begin{array}{l}\text { Características de la } \\
\text { interacción }\end{array}$ & $\begin{array}{l}\text { Las características de la interacción es que es compartidas a terceros, la cual al ser recibida } \\
\text { por parte de un familiar ésta posteriormente es replicada al resto de la familia extensa. Es } \\
\text { por ello la trascendencia de esta información ya que al interlocutor la replicará al resto de la } \\
\text { familia según la haya comprendido y es por ello que debe ser entregada de manera oportuna, } \\
\text { clara y comprensible. }\end{array}$ \\
\hline Comportamiento & $\begin{array}{l}\text { El comportamiento observado se caracteriza por ser heterogéneo. Se observan personas que } \\
\text { se encuentran nerviosas, con síntomas relacionados a esto, como náuseas, vómitos, por lo } \\
\text { que se deduce que a la unidad de urgencia asisten pacientes y familiares quienes presentar } \\
\text { una carga emocional importante relacionada más bien a emociones o sensaciones negativas, } \\
\text { ya sea de preocupación, ansiedad, nerviosismo, etc. }\end{array}$ \\
\hline Lenguaje no verbal & $\begin{array}{l}\text { Expresiones de malestar o dolor físico que presentan los pacientes lo manifiestan indicándolo } \\
\text { con sus manos, empelado para entregar información al equipo de salud sobre su patología, } \\
\text { vale decir, usuarios/as señala en qué sector de su cuerpo se concentra su dolor. }\end{array}$ \\
\hline Facilitadores de la atención & $\begin{array}{l}\text { La función del asistente social quien proporcionaba información tanto en la sala de urgencia } \\
\text { como en la sala de espera a familiares y pacientes, quien realiza un trabajo mancomunado } \\
\text { con el resto del equipo de salud enfocándose en la entrega de información. }\end{array}$ \\
\hline $\begin{array}{l}\text { Obstaculizadores de la } \\
\text { atención }\end{array}$ & $\begin{array}{l}\text { Restringida capacidad que presentan algunos/as pacientes para entregar información sobre su } \\
\text { estado de salud a los/as funcionarios/as de la salud, por lo cual éstos deben buscar distintas } \\
\text { alternativas para comprender el motivo de consulta del/la usuarios/a. }\end{array}$ \\
\hline Espacio físico & $\begin{array}{l}\text { Son todos aquellos espacios físicos que componen la urgencia, desde la puerta que da a la } \\
\text { sala de espera de la unidad de urgencia adulto y en la estación de enfermería de urgencia } \\
\text { pediátrica, lo cual transgrede la privacidad que debería caracterizar la información sobre el } \\
\text { estado de salud de un/a paciente. }\end{array}$ \\
\hline
\end{tabular}

Fuente: Elaboración propia a partir de cuatro momentos de observación (2019).

sarrollar competencias personales y profesionales de los/as funcionarios/as, fundamentales para la entrega adecuada de la información, como son: la empatía y el uso de un lenguaje sencillo y exento del uso de tecnicismos; ambos aspectos identificados como prioritarios desde la perspectiva de usuarios/as y familiares.

La teoría afirma que los medios o canales de información, tanto escritos como presenciales, deben estar a disposición de la población de manera simultánea, puesto que al acceder a información del estado de salud del/la usuario/a existen grados de estrés que obstaculizan la retención de toda la información, siendo olvidada con facilidad. También se debe tener competencias comunicativas personales y profesionales para el adecuado trato al usuario/a, considerando la heterogeneidad de la población y conocimiento de intervención en crisis, sobre todo frente el fallecimiento de usuarios/as y en esta investigación se ha constatado que no existe un protocolo que oriente al/la profesional en cómo entregar esta noticia a la familia.

Otro elemento que vulnera el derecho a la información de usuarios/as-familiares se atribuye al escaso tiempo que tiene el equipo de salud para entregar información, debido a la sobredemanda de la UUH. Por otro lado, la UUH cuenta con el servicio de enlace comunicacional, función ejercida por un Asistente Social, quien resulta ser el nexo entre el equipo de salud y usuarios/as-familiares, sin embargo, no está presente durante 
Tabla 2. Síntesis entrevistas a funcionarios/as

\begin{tabular}{|c|c|}
\hline & Síntesis de respuestas \\
\hline \multicolumn{2}{|l|}{ Lógica deductiva } \\
\hline $\begin{array}{l}\text { Percepción del funcionario/a frente } \\
\text { la entrega de información }\end{array}$ & $\begin{array}{l}\text { El contexto de la urgencia no es propicio para una entrega de información de cali- } \\
\text { dad. La entrega de información no es una prioridad en la urgencia. }\end{array}$ \\
\hline $\begin{array}{l}\text { Espacios para la entrega de } \\
\text { información }\end{array}$ & $\begin{array}{l}\text { No se cuenta con espacios físicos idóneos para entregar información a usuarios/as } \\
\text { y familiares, que conserven privacidad y confidencialidad. }\end{array}$ \\
\hline $\begin{array}{l}\text { Competencias personales y } \\
\text { profesionales }\end{array}$ & $\begin{array}{l}\text { Competencia personal primordial: La empatía. } \\
\text { Competencia profesional: Manejo en trato usuario/a considerando su heterogenei- } \\
\text { dad. Conocimiento de intervención en crisis. }\end{array}$ \\
\hline Medio o canal de comunicación & $\begin{array}{l}\text { La información escrita digitalizada facilita la comprensión del/la lector/a. La informa- } \\
\text { ción obtenida presencialmente no siempre es entendida por usuarios/as y familiares } \\
\text { cuando se emplean tecnicismos. }\end{array}$ \\
\hline Tiempo & $\begin{array}{l}\text { Tiempo precario para la entrega de información a usuarios/as y familiares. El enlace } \\
\text { comunicación no está presente durante todos los días y horas de la semana. }\end{array}$ \\
\hline Propuestas de mejoras & $\begin{array}{l}\text { Mejorar infraestructura que permita una adecuada entrega de información y capa- } \\
\text { citar al equipo de salud al respecto. }\end{array}$ \\
\hline \multicolumn{2}{|l|}{ Lógica inductiva } \\
\hline Sentimientos de usuarios/as & $\begin{array}{l}\text { Sentimientos catalogados como negativos, que tienen que ver con la ansiedad, el } \\
\text { enojo, la preocupación, frustración y nerviosismo, los cuales se agravan al no recibir } \\
\text { una información clara y oportuna por parte de los/as funcionarios/as de salud. }\end{array}$ \\
\hline Efectos en familiares & $\begin{array}{l}\text { Cuando la información que reciben familiares es básica o incompleta se puede gati- } \\
\text { llar nerviosismo, frustración, descontento con el servicio y puede generar conflictos } \\
\text { y a veces hasta agresión hacia los/as funcionarios/as. }\end{array}$ \\
\hline Efectos en funcionarios/as & $\begin{array}{l}\text { Si familiares no logran tener acceso a la información de su paciente se genera una } \\
\text { situación de conflicto con el equipo de salud. La intervención del asistente social } \\
\text { contribuye a proporcionar información a familiares ya que da tiempo para que los/ } \\
\text { as funcionarios/as se dediquen a realizar procedimientos clínicos, pero al mismo } \\
\text { tiempo el familiar logra acceder a la información. }\end{array}$ \\
\hline $\begin{array}{l}\text { Capacitación sobre entrega de } \\
\text { información }\end{array}$ & $\begin{array}{l}\text { Las capacitaciones sobre la entrega de información no son realizadas a funcionarios/ } \\
\text { as de la UUH y las reuniones técnicas o clínicas son escasas y las que se realizan no } \\
\text { abordan temáticas relacionadas a la entrega de información. }\end{array}$ \\
\hline $\begin{array}{l}\text { Nexo entre médico/a y } \\
\text { usuarios/as }\end{array}$ & $\begin{array}{l}\text { Funcionarios/as generan un nexo entre pacientes y médicos/as, vale decir, estos fun- } \\
\text { cionarios propician que exista una conversación entre paciente y médico/a cuando } \\
\text { el paciente solicita información sobre su diagnóstico. }\end{array}$ \\
\hline $\begin{array}{l}\text { Protocolo frente usuarios/as } \\
\text { fallecido }\end{array}$ & $\begin{array}{l}\text { No existe un protocolo que oriente cómo entregar esta información la que finalmen- } \\
\text { te es entregada a familiares según las habilidades de cada médico/a, quien es el/la } \\
\text { encargado/a de informar a familiares del fallecimiento de paciente. }\end{array}$ \\
\hline
\end{tabular}

Fuente: Elaboración propia a partir de cinco entrevistas a funcionarios/as (2019).

toda la semana y en todos los horarios, quedando desprovista durante la jornada de la tarde-noche, días festivos y los fines de semana.

Otra situación que vulnera el derecho a la información afecta a los/as familiares, quienes según los resultados de esta investigación se ven obligados a insistir en reiteradas ocasiones para acceder a información médica respecto de su familiar, lo que es percibido por los/as entre- vistados/as de este estudio como "inhumana y desagradable".

Se destaca el trato adecuado por parte de Guardias de Seguridad, TENS y Asistente Social quienes otorgaron un buen trato en la entrega de información. Un trato adecuado genera la sensación de alivio en el/la usuario/a-familiares, aun sintiendo un malestar físico intenso, propicia a que los/as usuarios/as esperen su turno serenamente, 


\section{Tabla 3. Síntesis resultados entrevistas a familiares}

\begin{tabular}{|c|c|}
\hline \multicolumn{2}{|l|}{ Lógica deductiva } \\
\hline $\begin{array}{l}\text { Percepción sobre la } \\
\text { atención en la UUH }\end{array}$ & $\begin{array}{l}\text { Hay percepciones positivas y negativas. La percepción positiva es frente la información recibida } \\
\text { por el guardia de seguridad y el asistente social. Quienes tienen una percepción negativa decla- } \\
\text { ran que la información fue "vaga y entregada de mala manera". }\end{array}$ \\
\hline Nivel de comprensión & $\begin{array}{l}\text { La información recibida es básica y no proporciona a los familiares mayores detalles del diag- } \\
\text { nóstico, tratamiento o pronóstico. Sí desean ser mayormente informados sobre el estado de } \\
\text { salud de su paciente y no sólo se enfoque a una categorización de estar bien, grave o agudo. }\end{array}$ \\
\hline Opinión sobre el acceso & $\begin{array}{l}\text { Para acceder a la información de su paciente deben insistir en reiteradas ocasiones su solicitud, } \\
\text { situación incómoda para ellos/as. En situaciones de fallecimientos los/as familiares manifiestan } \\
\text { disconformidad frente la información, por ser básica. }\end{array}$ \\
\hline Trato & $\begin{array}{l}\text { Se destaca positivamente el trato por parte de guardias de seguridad y TENS. Pero también hay } \\
\text { tratos hostiles en donde parte del equipo de salud no muestra disposición para atender a las } \\
\text { dudas de familiares. }\end{array}$ \\
\hline Medios o canales & $\begin{array}{l}\text { Los medios son presencial y escrito. Señalan que al tener a un ser querido en la UUH les genera } \\
\text { estrés, impidiendo que se retenga toda la información de su paciente y se olvide con facilidad. EI } \\
\text { documento escrito al que tienen acceso los familiares está elaborado en computador, evitando } \\
\text { que la letra manuscrita sea incomprendida para el/la lector/a. }\end{array}$ \\
\hline $\begin{array}{l}\text { Características de la } \\
\text { información }\end{array}$ & $\begin{array}{l}\text { Debe existir "tino" para entregar información, vale decir, empatía, sobre todo al informar fa- } \\
\text { llecimientos. Otro elemento es hacer sentir bien y cómodo, tener un trato amable que genere } \\
\text { un sentimiento de agrado que a pesar de los síntomas físicos que se presenten ya que palean } \\
\text { el malestar y generar un sentimiento de confort. }\end{array}$ \\
\hline \multicolumn{2}{|l|}{ Lógica inductiva } \\
\hline $\begin{array}{l}\text { Espacio físico donde } \\
\text { recibe información }\end{array}$ & $\begin{array}{l}\text { Mesones y pasillos de la UUH, lugares incómodos para familiares ya que no se respeta la priva- } \\
\text { cidad y confidencialidad de la información. }\end{array}$ \\
\hline Sentimiento/sensación & $\begin{array}{l}\text { El hecho de tener que insistir para acceder a la información del paciente es catalogado como } \\
\text { frustrante e inhumano. El recibir información de un diagnóstico delicado genera diversos sen- } \\
\text { timientos, los cuales pueden forjar inestabilidad emocionar aguda y que pueden decantar en } \\
\text { episodios críticos relacionados a la salud mental. }\end{array}$ \\
\hline
\end{tabular}

Fuente: Elaboración propia a partir de cinco entrevistas a familiares (2019).

disminuye la impaciencia y cuando perciben a los/ as funcionarios/as con disposición a responder inquietudes genera bienestar emocional. Por el contrario, recibir un trato inapropiado, hostil o ser testigo de ello, genera en el/la usuario/a-familiar malestar emocional.

\section{Conclusiones}

Como principal conclusión se puede aseverar que existe una vulneración importante en el derecho a la información de los usuarios/as en la UUH del HGGB, es decir, la entrega de información actualmente no cumple con los estándares establecidos en la normativa nacional relacionada a que la información sea suficiente, oportuna, veraz y comprensible.
Luego de la contrastación empírica, es posible señalar que los cinco supuestos preliminares de la investigación se confirman. Efectivamente la calidad de la información obtenida en la UUH en torno a diagnóstico, pronóstico y tratamiento presenta falencias relacionadas a su contenido, el que es poco claro y utiliza tecnicismos escasamente comprendidos por los usuarios y familiares, siendo una información más bien somera. Todo lo anterior, no permite que la información entregada sea conforme a los estándares establecidos en la Ley 20.584 .

Los resultados de esta investigación dan cuenta diversas brechas observadas para garantizar la entrega de información suficiente, oportuna, veraz y comprensible a los usuarios-familiares, considerando: 
Tabla 4. Síntesis entrevistas a usuarios/as

Nivel de comprensión Se logra a través de un lenguaje sencillo, exento del uso de tecnicismos, los cuales tienden a

Opinión sobre el acceso Se valora el acceso a la información cuando es el/la médico/a quien se encarga de entregar la

Existe una percepción positiva y negativa. Es positiva cuando es el/la médico/a quien entrega información expedita y completa sobre el estado de salud y negativa cuando se entrega una información somera, en donde no se explica a cabalidad la información del diagnóstico, pronóstico y tratamiento. insegurizar y confundir al/la usuario/a. Un elemento importante de destacar es el uso de internet por parte de usuarios/as, el cual resulta ser una herramienta clarificadora en torno a tratamientos y medicamentos, sobre todo si son complejos, la cual tiene la particularidad de ser expedita y de fácil acceso para todos/as. información de manera personalizada, lo que es importante y necesario. Caso contrario ocurre cuando es el/la paciente quien buscaba al/la médico/a para obtener información y que la calidad de ésta dependerá de sus habilidades blandas, siendo algunos detallistas y otros reservados, vale decir que no profundizan en toda la información.

Trato

Al recibir un buen trato se genera la sensación de alivio en el paciente, aun sintiendo un malestar físico intenso. El buen trato propicia a que los/as pacientes esperen su turno serenamente y se evita su impaciencia. Percibir a funcionarios/as con disposición a responder contribuye a que se sienta mejor emocionalmente. Pero, recibir un trato inapropiado, hostil o ser testigo de uno genera en el paciente malestar emocional.

Medios o canales

Tanto la vía oral como escrita son importantes y se complementan. El traspaso de información presencial requiere la utilización de un lenguaje de fácil comprensión y que esté exento de tecnicismos y el escrito es útil para respaldar la información que entrega el/la médico/a y así volver a revisar por si se olvida su contenido o se generan dudas.

Características de la Se utilizaron adjetivos positivos como claro, rápido, explícito, concreto, asertivo y adjetivos neinformación gativos como técnico, médico, redundante, términos elevados de difícil comprensión y escasa empatía al entregar información.

\section{Lógica inductiva}

Propuestas de Mejoras

Utilización de un lenguaje más sencillo y de fácil comprensión para todos/as los/as usuarios/as y que el traspaso de información vaya unida a un buen trato. No sólo es importante recibir toda la información relacionada con el estado de la salud, sino que recibirla de una manera cordial.

Sentimiento/sensación

La persona al recibir una noticia de un diagnóstico complejo puede impresionarse a tal punto que se llega a paralizar. El no poder acceder a la información sobre el estado de salud puede ser frustrante, hasta humillante lo cual genera disconformidad y episodios agresivos por parte de pacientes.
\end{abstract}

Fuente: Elaboración propia a partir de cinco entrevistas a usuarios/as (2019).

- Competencias del Equipo Salud: Es necesaria la presencia de competencias personales como empatía y cordialidad, pero también competencias profesionales como manejo en intervención en crisis, y potenciar las habilidades comunicativas relacionadas al trato al/ el usuario/a-familiar.

- Tiempo para entrega información: Por la constante sobredemanda de la UUH el equipo de salud no cuenta con el tiempo suficiente para informar a usuarios/as- familiares en profundidad. Canales de comunicación:
Para que la información sea comprendida adecuadamente debe ser proporcionada de manera oral y escrita simultáneamente al/a usuario/a-familiar, de lo contrario interfiere en la comprensión total, sin embargo en la actualidad no es así.

- Espacio físico: No existe un espacio físico que garanticen la confidencialidad y privacidad en la entrega de información a usuarios/as y familiares, actualmente se realiza en espacios comunes, como pasillos, salas de espera etc.

- Continuidad de la atención de Enlace Comu- 


\section{Tabla 5. Integrado de resultados por categorías centrales*}

Percepción frente la entrega de información

Espacios para la entrega de información

Competencias personales y profesionales

Medio o canal de comunicación

Tiempo

El contexto de la urgencia no es el propicio para entregar la información adecuada. Entregar información no es una prioridad. Para que sea de calidad debe ser detallada y completa, no obstante, también es entregada de manera somera, en donde no se explica a cabalidad la información del diagnóstico, pronóstico y tratamiento.

No se cuenta con espacios físicos idóneos para entregar información a usuarios/as y familiares que conserven privacidad y confidencialidad, los utilizados son mesones, pasillos, sala de espera, al lado de la camilla, sector de hidratación. Se creó una oficina para entregar información la cual debido a la saturación de la unidad es utilizada para hospitalizaciones.

Competencia personal primordial: La empatía.

Competencia profesional: Manejo en trato usuario/a considerando su heterogeneidad. Conocimiento de intervención en crisis.

Son presencial y escrito. Al acceder a información del estado de salud del paciente existen grados de estrés que obstaculizan la retención de toda la información de diagnóstico, pronóstico y tratamiento y se olvide con facilidad. El documento escrito al está elaborado en computador, evitando que la letra manuscrita sea incomprendida para el/la lector/a.

Tiempo precario para la entrega de información a usuarios/as y familiares. El enlace comunicacional no está presente durante todos los días y horas de la semana.

Propuestas de mejora Mejorar infraestructura que permita una adecuada entrega de información y capacitar al equipo de salud al respecto.

Características de la información

Se utilizaron adjetivos positivos como clara, rápida, explícita, concreta, asertiva y negativos como técnica, médica, redundante, términos elevados de difícil comprensión y escasa empatía al entregar información.

Opinión sobre el acceso Para acceder a la información médica se deben insistir en reiteradas ocasiones su solicitud, situación incómoda tanto para usuarios/as como para familiares. En situaciones de fallecimientos los/as familiares manifiestan disconformidad frente la información, por ser básica y no existir contención frente la noticia.

Trato

Se destaca el trato adecuado de Guardias de Seguridad, TENS y asistente social. Un buen trato genera la sensación de alivio en el paciente, aun sintiendo un malestar físico intenso, propicia a que los/as pacientes esperen su turno serenamente y se evita su impaciencia, percibir a funcionarios/as con disposición a responder inquietudes contribuye a que se sienta mejor emocionalmente. Pero, recibir un trato inapropiado, hostil o ser testigo de uno genera en el paciente $y / 0$ familiar malestar emocional.

Nivel de comprensión

Se logra a través de un lenguaje sencillo, exento del uso de tecnicismos, los cuales tienden a insegurizar y confundir al/la usuario/a. El uso de internet por parte de usuarios/as resulta ser una herramienta de apoyo y comprensión de la información, de fácil acceso para todos/as.

* El cuadro 5 sintetiza los resultados arrojados por las entrevistas realizadas a funcionarios/as, familiares y usuarios/as. Fuente: Elaboración propia a partir de 15 entrevistas realizadas a funcionarios/as, familiares y usuarios/as (2019).

nicacional: Existe sólo un profesional asistente social que cumple la función de enlace comunicacional, cuyo horario de funcionamiento es de lunes a viernes de 08:00 a 17:00 hrs, quedando desprovista la UUH de su enlace comunicacional en beneficio a familiares y como apoyo al mismo equipo de salud, luego del horario señalado y fines de semana y días festivos.
- Déficit de Protocolos de Actuación: Actualmente la UUH no cuenta con protocolo para informar fallecimiento de usuarios/as a sus familiares, por las características de esta situación y el impacto emocional que implica, se requiere disponer de un procedimiento regulado, evitando consecuencias negativas para las familias. 
Tabla 6. Respuesta a la pregunta central de investigación*

Pregunta de investigación: ¿La información otorgada sobre diagnóstico y tratamiento a los usuarios/as de las unidades de urgencia hospitalaria cumple con los estándares del derecho a la información establecidos en la Ley 20.584?

En sus artículos del 8 al 11 mencionan que se debe emplear un lenguaje adecuado e inteligible al acceder a la información sobre el estado de salud del/la usuario/a, resguardando la privacidad y la honra de éste/a mientras se mantenga este diálogo. Mientras que en Artículo 10 se señala que específicamente que toda persona tiene derecho a ser informada, en forma oportuna y comprensible, por parte del/la médico/a u otro profesional tratante.

En el párrafo 4 Del Derecho a la Información se indica que decir la información debe cumplir con ciertas características como ser suficiente, oportuna, veraz y comprensible, la que puede entregarse de manera visual, verbal o por escrito.

La información que debe entregar el médico u otro profesional tratante debe ser relativa a estado de salud, posible diagnóstico, alternativas de tratamiento, riesgos que ello pueda representar, pronóstico esperado y proceso previsible del posoperatorio cuando procediere.

Cuando la condición de la persona, a juicio de su médico tratante, no le permita recibir la información directamente o padezca de dificultades de entendimiento o se encuentre con alteración de conciencia, la información será dada a su representante legal, o en su defecto, a la persona bajo cuyo cuidado se encuentre.

Los prestadores deberán adoptar las medidas necesarias para asegurar la adecuada confidencialidad durante la entrega de esta información, así como la existencia de lugares apropiados para ello.
No es constante una entrega de información adecuada e inteligible, ya que está supeditada a factores como el contexto, tiempo y competencias profesionales y personales de cada funcionario/a de la salud. La privacidad de la información no es garantizada, debido a las características propias de la urgencia, en donde no se cuenta con un espacio físico idóneo. Cuando el/la médico/a no puede informar, lo deben hacer otros funcionarios/as, no obstante están limitados/as para entregar toda la información de salud, por lo que deben proceder a hacer un "enganche" entre éste/a y usuarios/as- familiares para que se logre el acceso a la información, el cual no siempre se consigue.

Si bien la información se entrega de manera verbal y escrita, las características que se le atribuyeron durante esta investigación no son todas positivas como asertiva y oportuna, sino que se cataloga como fue vaga, inconclusa, técnica, médica, redundante y escasa empatía al entregar información.

La información recibida es básica y no proporciona a los familiares mayores detalles del diagnóstico, tratamiento o pronóstico. Pacientes y familiares demandan mayor información sobre el estado de salud y no que ésta se reduzca a una categorización de estar "bien, grave o agudo", como se devela en esta investigación.

En general ese tercero es un familiar y/o referente significativo, quienes según lo develado en esta investigación son los más desprovistos de información, incumpliendo este apartado de la ley.

La UUH de HGGB no cuenta con espacios físicos idóneos para la entrega de información según lo estipulado en esta ley, vulnerando así el derecho a la información con el componente de confidencialidad, lo cual es urgente de resolver para garantizar el derecho a la información de usuarios/as.

*El cuadro 6 sintetiza los resultados arrojados en esta investigación que dan respuesta a la pregunta de investigación. Fuente: Elaboración propia a partir de 15 entrevistas realizadas a funcionarios/as, familiares y usuarios/as (2019).

A partir de las brechas identificadas, se plantean las siguientes Propuestas de Mejora:

- Fortalecer las competencias profesionales y personales del equipo de salud, como manejo de intervención en crisis, habilidades comunicativas y empatía.

- Diseñar e implementar un Protocolo de Actuación para informar el fallecimiento de usuarios/as a sus familiares.
- Considerar en la programación el tiempo suficiente para la entrega de información en torno al diagnóstico, tratamiento y pronóstico a usuarios/as y familiares.

- Realizar las gestiones necesarias para que los equipos de salud puedan entregar información por el canal oral y escrito en forma simultánea, para permitir que sea comprendida adecuadamente. 
Tabla 7. Acceso a la información de usuarios/as y familiares*

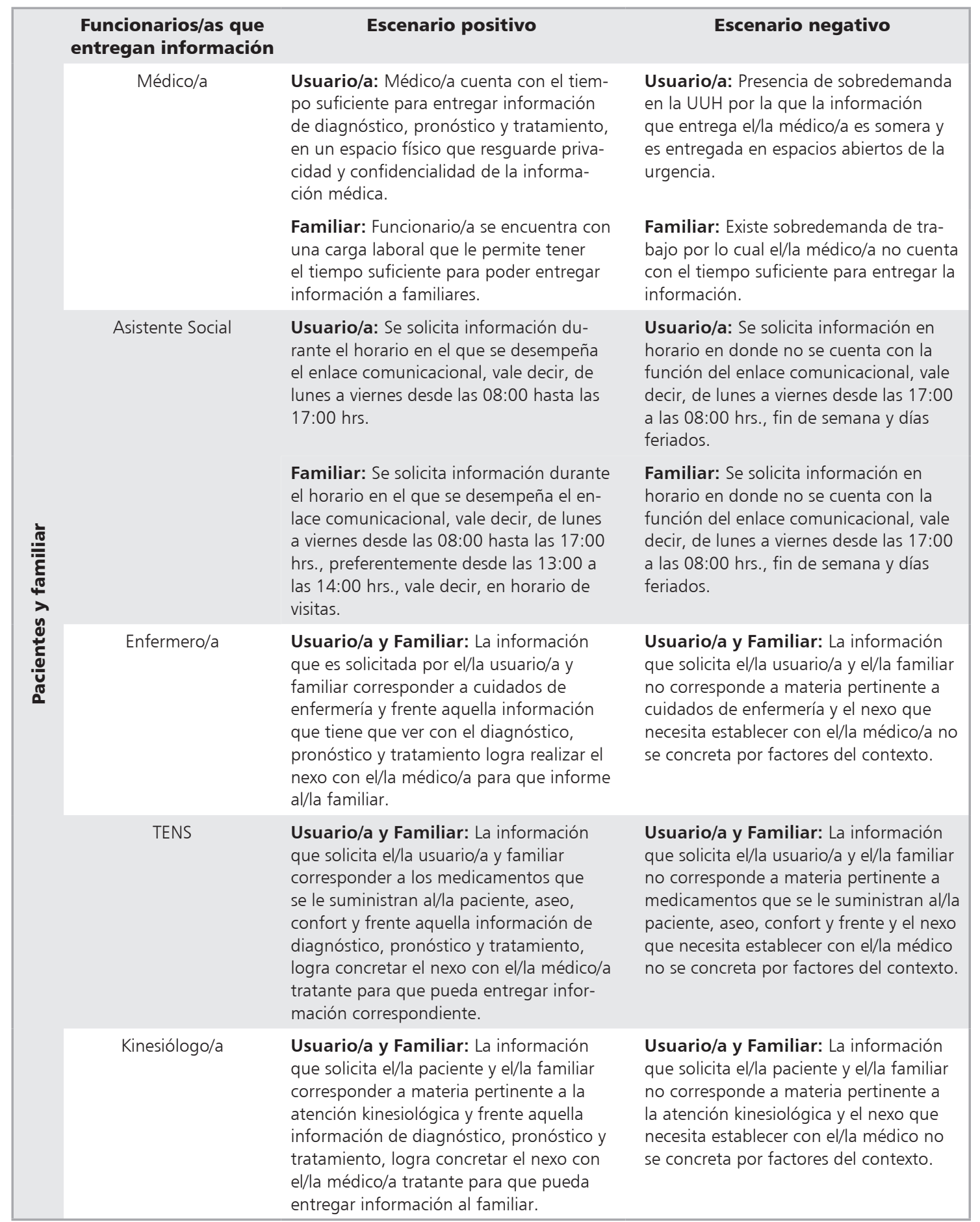

*El cuadro 7 sintetiza los resultados arrojados, identificando a los/as distintos/as funcionarios/as que entregan información a usuarios/as y familiares, en escenarios tanto positivos como negativos. Fuente: Elaboración propia a partir de 15 entrevistas realizadas a funcionarios/as, familiares y usuarios/as (2019). 


\section{Tabla 8. Espacios donde se entrega información a usuarios/as y familiares por parte de funcionarios/as de la salud*}

\begin{tabular}{|c|c|}
\hline Espacios & Características de los espacios \\
\hline Mesones de la UHH & $\begin{array}{l}\text { Constantemente están presente los/as funcionarios/as que se desempeñan en la urgencia, } \\
\text { no sólo aquellos que está entregando el servicio al paciente. } \\
\text { Presencia de personas ajenas al paciente. No propicia las condiciones para la entrega de } \\
\text { información de manera cómoda y privada para el/la familiar. }\end{array}$ \\
\hline Sector de hidratación & $\begin{array}{l}\text { Se trata de un box amplio, equipado con sillas y sillones en donde existe presencia de } \\
\text { pacientes que se encuentran en proceso de suministro de suero y medicamentos. }\end{array}$ \\
\hline Pasillos & $\begin{array}{l}\text { Espacios que conectan la UHH, los cuales cuenta con constante afluencia de funcionarios/ } \\
\text { as, familiares, pacientes y hospitalizados. }\end{array}$ \\
\hline $\begin{array}{l}\text { Sala de espera Urgencia adulto } \\
\text { y Pediatría }\end{array}$ & $\begin{array}{l}\text { Presencia de pacientes esperando atención médica y público en general. Espacio abierto, } \\
\text { equipado con sillas en donde funcionarios/as entregan información a familiares desde la } \\
\text { puerta de ingreso a la urgencia. }\end{array}$ \\
\hline Boxs & $\begin{array}{l}\text { Son espacios cerrados en donde existe mobiliario apropiado para la atención de pacientes } \\
\text { tal como mesón, camilla, sillas y computador para atender al paciente. Favorece la comu- } \\
\text { nicación entre funcionarios/as y pacientes de forma privada. }\end{array}$ \\
\hline Oficina entrevista Familiar & $\begin{array}{l}\text { Oficina utilizada principalmente para generar hospitalizaciones a pacientes con diagnós- } \\
\text { tico crítico. Cuenta con una camilla, mesón, sillas y un computador. En primera instancia } \\
\text { fue creada para la entrega de información a pacientes y familiares, no obstante, debido } \\
\text { a la sobredemanda de la urgencia, en la actualidad es utilizada para hospitalizaciones. }\end{array}$ \\
\hline Sector camillas & $\begin{array}{l}\text { Una cortina (biombo) separa una camilla de otra, en donde el/la paciente es retroalimen- } \\
\text { tado con información sobre su diagnóstico, pronóstico y tratamiento. }\end{array}$ \\
\hline
\end{tabular}

*El cuadro 8 identifica los distintos espacios de la UUH utilizados para entregar información y sus características. Fuente: Elaboración propia a partir de 15 entrevistas realizadas a funcionarios/as, familiares y usuarios/as (2019).

\section{Tabla 9. Medios o canales utilizados para entrega información a usuarios/as y familiares*}

\section{Presencial}

El acceso de información de manera presencial es el más valorado tanto por usuarios/as como por familiares.

Es valorado porque el/la usuarios/a y/o familiar se validado al tener conocimiento de que el/la médico/a tratante dispuso de tiempo para entregar información y resolver dudas.

Éste se puede establecer en los espacios anteriormente descritos, siendo el más valorado por los/as entrevistados/as la oficina, en donde el/la médico/a tratante entregua la información.

Las características de este medio-canal debe ser con un leguaje sencillo, simple, exento de tecnicismos, que le permitan al/la interlocutor/a comprender adecuadamente la información médica a la que está accediendo.

\section{Escrita}

El medio-canal escrito resulta ser un apoyo significativo al momento de acceder a la información, valorado tanto por usuarios/ as como familiares, puesto que se configura como un "apoyo memoria" posterior a la atención de salud en la urgencia.

Los medios-canales más valorados por usuarios/as y familiares son los exámenes médicos y documento de Datos de Atención de Urgencia (DAU).

Un factor positivo de DAU es que desde el año 2018 es elaborado digitalmente, facilitando la comprensión de la información por parte del/la lector/a, ya que anteriormente era entregado con letra manuscrita.

Se develó que una herramienta utilizada para la comprensión de la información es el Internet, al cual usuarios/as y familiares acceden para consultar respecto a medicamentos alternativos (más económicos que los recetados) para indagar sobre algún diagnóstico específico, causas-consecuencias y para volver a consultar sobre diagnóstico, pronóstico y tratamiento cuando la información recibida por parte de equipo de salud se olvidó, posterior al alta médica.

*El cuadro 9 señala medios y canales utilizados para entregar información a usuarios y familiares en la UUH. Fuente: Elaboración propia a partir de 15 entrevistas realizadas a funcionarios/as, familiares y usuarios/as (2019). 
- Disponer de un espacio físico que garantice la confidencialidad y privacidad en la entrega de información a usuarios/as y familiares; con condiciones de orden, limpieza y comodidad.

- Ampliar la dotación de profesionales en el rol de enlace comunicacional, y ampliar los horarios y días acorde al funcionamiento de la UUH.

Agradecimientos: Se agradece el imprescindible trabajo colaborativo por parte de los Directivos del Hospital Guillermo Grant Benavente, y en especial del jefe de la Unidad de Urgencia Hospitalaria, quienes permitieron que la presente investigación se llevara a cabo en dicho establecimiento de salud. Al mismo tiempo se agradece y destaca el profesionalismo del Asistente Social de la Unidad de Urgencia, quien otorgó orientación y asesoría a la investigadora en las diversas fases del proceso.

\section{Refererencias}

1. Intituto Nacional de Derechos Humanos [INDH]. (2016). Informe anual situación de los Derechos Humanos en Chile. Territorios y Derechos Humanos. Obtenido de Biblioteca digital INDH: https://bibliotecadigital. indh.cl/handle/123456789/998.

2. Organización para la Cooperación y el Desarrollo económico [OCDE](2014). Estadísticas de la OCDE sobre la salud 2014 Chile en comparación. Obtenido de oecd.org: https://www.oecd.org/els/health-systems/ Briefing-Note-CHILE-2014-in-Spanish.pdf.

3. Organización Mundial de la Salud [OMS] (29 de diciembre de 2017). Salud y Derechos Humanos. Obtenido de Organización Mundial de la Salud : www. who.int/es/news-room/fact-sheets/detail/human-rights-and-health.

4. MINSAL (2018). Orientaciones Técnicas para el Rediseño al Proceso de Atención de Urgencia de Adulto, en las Unidades de Emergencia Hospitalaria. Obtenido de MINSAL: https://www.minsal.cl/wp-content/ uploads/2018/03/Redise\%C3\%B1o-al-proceso-de-atenci\%C3\%B3n-de-urgencia-de-adulto-en-las-unidades-emergencia-hospitalaria.pdf.

5. Biblioteca del Congreso Nacional [BCN] (24 de mayo de 2012). Historia de la Ley $N^{\circ} 20.584$. Obtenido de BCN: https://www.bcn.cl/historiadelaley/nc/historia-de-laley/4579/.

6. Tojar C. (2006). La Investigación Cualitativa: comprender y actuar. Madrid: La Muralla.

7. MINSAL (2019). Hospital Guillermo Grant Benavente destacó en medición latinoamericana sobre recintos mejor equipados, obtenido de www.minsal.cl/hospital-guillermo-grant-destaco-en-medicion-latinoamericana-sobre-recintos-mejor-equipados.

8. Verd J, Lozares C. (2016). Introducción a la Invetsigación Cualitativa. Madrid: Síntesis

9. Ley $\mathrm{N}^{\circ}$ 20.584. Diario Oficial de la República de Chile, Santiago, Chile, 24 de abril 2012. https://www.bcn.cl/ leychile/navegar?idNorma $=1039348$. 